\title{
Semi-Analytical Solution for Formation Flying Spacecraft Subject to Electromagnetic Acceleration
}

\author{
S. K. Tealib ${ }^{1, *}$, Yehia Abdel-Aziz ${ }^{1}$, M. E. Awad ${ }^{2}$, Kh. I. Khalil ${ }^{1}$, M. Radwan ${ }^{2}$ \\ ${ }^{1}$ National Research Institute of Astronomy and Geophysics (NRIAG), Egypt \\ ${ }^{2}$ Faculty of Science, Cairo University, Egypt
}

Received November 8, 2019; Revised December 23, 2019; Accepted December 25, 2019

Copyright $\bigcirc 2020$ by authors, all rights reserved. Authors agree that this article remains permanently open access under the terms of the Creative Commons Attribution License 4.0 International License

\begin{abstract}
The generation of Lorentz force is limited by the local magnetic field and the relative velocity of the spacecraft. As a result of this constraint, the Lorentz force cannot completely take place of the traditional propulsion technologies. In recent years, studying the Lorentz force provided by modulating spacecraft electrostatic charge (magnetic and electric fields) has become a possible good means to control the spacecraft without fuel consumption or reduce the fuel cost. Most of the previous studies concerned the Lorentz force of the magnetic field only. In this paper, we developed a combination of Lorentz forces provided by modulating spacecraft's electrostatic charge (magnetic and electric fields) that can be used to keep the desired relative distances and orientations. We are derived nonlinear dynamic models of satellite relative motion considering $\mathrm{J}_{2}$ perturbation, and Lorentz forces to develop a mathematical model for a new technique establishing increase the level of charging in the spacecraft surface. These solutions indicate that the principal effects of the Lorentz force on a spacecraft can be used to correct the drift in the relative position of formation flying due to the effect of Second zonal harmonics, where the magnitude of charge to mass ratio is the key player for the correction of this drift. Numerical results for different examples of formation flying confirmed the capability of Lorentz force to correct the drift in relative position. These corrections depend on the value of charge to mass ratio and magnitude of the relative position.
\end{abstract}

Keywords Lorentz-force, Electrostatic Charge, Nonlinear Relative Motion, Lorentz Force

\section{Introduction}

Spacecraft charging occurred during the orbital motion of the spacecraft in the space plasma and charged particle moving relative to Earth's magnetic field in the direction perpendicular to its velocity, creating a current along its orbital path. The main goal to maintain an electrostatic charge on the surface of the spacecraft, creating Lorentz force due to the interaction between the local magnetic field and the velocity of the spacecraft, by developing the effect of Lorentz force for both magnetic and electric fields to increase the level of charging on the spacecraft surface using Ion collector. Nonlinear equations for spacecraft formation flying under $\mathrm{J}_{2}$ perturbation have been used extensively in this field (B. Kuiack and S. Ulrich [1]). M. Bakhtiari et al. [2] developed Lagrangian equations of formation flying considering $\mathrm{J}_{2}$ perturbation and showed that the main body's obliquity has a remarkable effect on formation keeping in the examined in-track. Early studies of spacecraft charging conclude that the Natural spacecraft charging level may reach about $10^{-8} \mathrm{C} / \mathrm{kg}$ (Vokrouhlicky 1989). Due to these limitations, the Lorentz force with such charging level is insufficient to perturb the orbit or attitude of satellite significantly, where the charge to mass ratio required to the Lorentz force for orbital maneuvering in low-Earth orbit (LEO) about $10^{-5} \mathrm{C} / \mathrm{kg}$ (M. Peck[4]). M. Peck [4] Proposed a new concept of active application of charge a spacecraft, this mechanism generates a net charge on the surface of spacecraft to induce Lorentz force via interaction with the Earth's magnetic fields. Y.A. AbdelAziz [5], Y.A. Abdel-Aziz, and K.I. Khalil [6] studied the effects of a Lorentz force on the orbital motion in Low Earth Orbit (LEO) and developed a model for the effects of electromagnetic forces (Lorentz force) to modify or perturb the spacecraft orbits, using Lagrange's planetary equations in Gauss form. G.E. Pollock et al. [7] developed the relative motion of a charged spacecraft under perturbations from the Lorentz force through interactions with the planetary magnetosphere. S. Tsujii et al. [8] derived a mathematical model of a charged satellite considering the effect of Lorentz force, for elliptical and circular cases of formation flying satellite orbit. X. Huang et al. [9] studied the analytical expressions for the orbital motion of Lorentz spacecraft with respect to inclined low Earth orbit and 
using the information from line-of-sight observations and gyro measurements, and designed both extended and unscented Kalman filter to perform relative navigation for Lorentz spacecraft. C. Peng, and Y. Gao [10] investigated a nonlinear dynamical model for relative motion assuming that the first satellite generates a rotating magnetic dipole while a constantly charging second satellite moves close to the artificial magnetic field of the chief satellite.

In this work, we derived a solution of the relative motion of Lorentz spacecraft for both magnetic and electric fields considering a small device (Ion collector) on the spacecraft to increase the level of charging on the spacecraft surface. Then, algorithms are designed to estimate the required value of the charge-to-mass ratio to correct the drift in the relative position due to the effect of second zonal harmonics perturbation. Finally, the numerical results for different examples of formation satellites are simulated to clarify the magnitude of charge-to-mass ratio for each example.

\section{Nonlinear Relative $\mathbf{J}_{\mathbf{2}}$ Perturbations}

Due to the limitation of the Cartesian coordinate system, we use a nonlinear relative dynamics model under the effect of $J_{2}$ perturbation. The reader is referred to as G. Y. $\mathrm{Xu}$, and D. W. Wang [11] for further details of derivatives of nonlinear dynamics model. Firstly, a local-verticallocal-horizontal (LVLH) frame attached to the chief satellite. This coordinate frame rotates with the chief's radius vector and is a convenient reference frame to describe the relative motion, the $\mathrm{x}$-axis (radial direction) is directed from center of the Earth towards the chief satellite, and the z-axis (cross-track) lies in the direction of the chief's orbital angular momentum, and the $y$-axis (along-track) completes the right-handed orthogonal triad M. Bakhtiari et al. [2].

The position of the deputy satellite is given by H.D. Curtis [12]

$$
\mathbf{r}_{d}=\mathbf{r}+\boldsymbol{\rho}=\left(r_{d}+x\right) \hat{i}+y \hat{j}+z \hat{k}
$$

where $\mathbf{r}_{d} \& \mathbf{r}$ are the position vectors for the deputy and chief satellites and $\boldsymbol{\rho}$ is the relative position vector between the deputy and the chief satellites.

The nonlinear equations of relative motion can be described as follows G. Y. Xu, and D. W. Wang [11]

$$
\begin{gathered}
\ddot{\boldsymbol{\rho}}=\left[\begin{array}{ll}
\ddot{\mathrm{x}} \quad \ddot{\mathrm{y}} \quad \ddot{\mathrm{z}}
\end{array}\right]^{\mathrm{T}}=\mathbf{a}(\boldsymbol{\rho}, \dot{\boldsymbol{\rho}})+\mathbf{a}_{\mathrm{J}}+\mathbf{a}_{\mathrm{L}} \\
\mathbf{a}(\boldsymbol{\rho}, \dot{\boldsymbol{\rho}})=\left[\begin{array}{c}
2 \dot{\theta} \dot{y}+\ddot{\theta} y+\dot{\theta}^{2} x+\frac{\mu}{r^{2}}-\frac{\mu}{r_{d}{ }^{3}}(x+r) \\
-2 \dot{\theta} \dot{x}-\ddot{\theta} x+\dot{\theta}^{2} y-\frac{\mu}{r_{d}{ }^{3}} y \\
-\frac{\mu}{r_{d}{ }^{3}} z
\end{array}\right]
\end{gathered}
$$

where $\mu$ is the gravitational parameter, $\dot{\boldsymbol{\theta}}=\frac{\mathbf{r} \times \dot{\mathbf{r}}}{r^{2}}$ is the angular rotational velocity vector, and the angular acceleration vector $\ddot{\boldsymbol{\theta}}=-2 \frac{\mathbf{r} \cdot \dot{\mathbf{r}}}{r^{2}} \dot{\boldsymbol{\theta}}$ H.D. Curtis [12]

$\mathbf{a}_{J}=\left[\begin{array}{lll}a_{J x} & a_{J y} & a_{J z}\end{array}\right]^{T}$ is the second zonal harmonic acceleration, and $\mathbf{a}_{L}=\left[\begin{array}{lll}a_{L x} & a_{L y} & a_{L z}\end{array}\right]^{T}$ is the total Lorentz acceleration.

The gravitational potential energy of chief satellite can be written as J.S. Ginn [13]

$$
\mathbf{U}=-\frac{\mu}{r}-\frac{3 \mu J_{2} R_{e}^{2}}{2 r^{3}}\left(\frac{1}{3}-Z^{2} / r^{2}\right)
$$

The gradient of $\mathrm{U}$ in Eq. (4) is computed in the LVLH frame to be J.S. Ginn [13]

$$
\nabla \mathbf{U}=\frac{\mu}{r^{2}} \hat{x}+\frac{3 \mu J_{2} R_{e}^{2}}{2 r^{4}}\left\{\begin{array}{c}
1-3 \sin ^{2} i \sin ^{2} \theta \\
2 \sin ^{2} i \sin 2 \theta \\
\sin 2 i \sin \theta
\end{array}\right\}\left[\begin{array}{l}
\hat{x} \\
\hat{y} \\
\hat{z}
\end{array}\right]
$$

The $\mathrm{J}_{2}$ nonlinear differential equations describing the relative motion of a charging spacecraft are:

$$
\begin{aligned}
\ddot{x}= & 2 \dot{\theta}_{z} \dot{y}+\ddot{\theta}_{z} y+\dot{\theta}_{z}{ }^{2} x-\xi_{d} x-\dot{\theta}_{x} \dot{\theta}_{z} z \\
& -\left(\zeta_{d}-\zeta\right) \sin i \sin \theta-r\left(\xi_{d}-\xi\right)+a_{L x}\left(\frac{\pi}{2}-\theta\right) \\
\ddot{y}= & -2 \dot{\theta}_{z} \dot{x}+2 \dot{\theta}_{z} \dot{z}-\ddot{\theta}_{z} x+\left(\xi_{d}-\dot{\theta}_{z}{ }^{2}-\dot{\theta}_{x}{ }^{2}\right) y+\ddot{\theta}_{x} z \\
& -\left(\zeta_{d}-\zeta\right) \sin i \cos \theta+a_{L y} \\
\ddot{z}= & -2 \dot{\theta}_{x} \dot{y}-\dot{\theta}_{x} \dot{\theta}_{z} x-\ddot{\theta}_{x} y- \\
& \left(\xi_{d}-\dot{\theta}_{x}{ }^{2}\right) z-\left(\zeta_{d}-\zeta\right) \cos i+a_{L z}
\end{aligned}
$$

where

$$
\begin{gathered}
\zeta=\frac{3 \mu J_{2} R_{e}^{2}}{r^{4}} \sin i \sin \theta, \\
\xi=\frac{\mu}{r^{3}}+\frac{3 \mu J_{2} R_{e}^{2}}{2 r^{5}}-\frac{15 \mu J_{2} R_{e}^{2}}{2 r^{5}} \sin ^{2} i \sin ^{2} \theta \\
\zeta_{d}=\frac{3 \mu J_{2} R_{e}^{2}}{r_{d}^{5}} \mathbf{r}_{d z}, \\
\xi_{d}=\frac{\mu}{r_{d}^{3}}+\frac{3 \mu J_{2} R_{e}^{2}}{2 r_{d}^{5}}-\frac{15 \mu J_{2} R_{e}^{2}}{2 r_{d}^{7}} \mathbf{r}_{d z}, \\
\mathbf{r}_{d z}=(r+x) \sin i \sin \theta+y \sin i \cos \theta+z \cos i \\
\dot{\theta}_{x}=-\frac{3 \mu J_{2} R_{e}^{2}}{2 h r^{3}} \sin 2 i \sin \theta, \quad \dot{\theta}_{z}=\frac{h}{r^{2}} \\
\ddot{\theta}_{z}=-\frac{2 h \dot{r}}{r^{3}}-\frac{3 \mu J_{2} R_{e}^{2}}{r^{5}} \sin ^{2} i \sin 2 \theta
\end{gathered}
$$




$$
\begin{aligned}
\ddot{\theta}_{x} & =-\frac{3 \mu J_{2} R_{e}^{2}}{2 r^{5}} \sin 2 i \cos \theta \\
& +\frac{9 \mu J_{2} R_{e}^{2}}{2 r^{4} h} \sin 2 i \cos \theta \\
& -\frac{36 \mu^{2} J_{2}^{2} R_{e}^{4}}{r^{6} h^{2}} \sin ^{2} i \cos i \sin ^{2} \theta \cos \theta
\end{aligned}
$$

$\theta \& h \& i$ are the true anomaly, norm angular momentum, and inclination of the chief satellite.

\section{Electromagnetic Force (Lorentz Force)}

The Lorentz force induced by an ambient space plasma and magnetic field $\mathbf{B}$ on charged particles with a velocity $\mathbf{v}_{\mathrm{r}}$ with respect to that field can be written as F.T.Ulaby [14]

$$
\begin{aligned}
\mathbf{F}_{L} & =\mathbf{F}_{m}+\mathbf{F}_{e} \\
& =q\left(\mathbf{v}_{\mathbf{r}} \times B\right)+q \mathbf{E}=q\left[\mathbf{v}_{\mathbf{r}} \times B+\mathbf{E}\right]
\end{aligned}
$$

Where, magnetic force $\left(\mathbf{F}_{\mathrm{m}}\right)$ is always perpendicular to the magnetic field and experienced by a particle of total electrostatic charge q (Coulombs) moving through a magnetic field $\mathbf{B}$, however, the electric force $\left(\mathbf{F}_{\mathrm{e}}\right)$ always in the direction of the electric field, acts on a charged particle whether or not it is moving, and $\mathbf{E}$ is an electric field.

\subsection{Lorentz Force Experience with Magnetic Field}

Assume that the magnetic dipole is not tilted such that the field lines near the equatorial plane are in the $+\hat{z}$ direction. The magnetic field can be expressed as L.U. Si-Zhen [15]:

$$
\begin{aligned}
\mathbf{B}= & {\left[\begin{array}{lll}
B_{r} & B_{\Theta} & B_{\phi}
\end{array}\right]^{T} } \\
& =\frac{B_{0}}{r^{3}}[2 \cos \Theta \hat{\mathbf{r}}+\sin \Theta \hat{\Theta}+0 \hat{\phi}]
\end{aligned}
$$

where $\mathrm{B}_{0}$ is the magnetic dipole moment of Earth $\left(8 \times 10^{15}\right.$ $\mathrm{T} \mathrm{m}^{3}$ ).

The magnetic field in the inertial (ECI) frame given by:

$$
\mathbf{B}=\left[\begin{array}{lll}
B_{x} & B_{y} & B_{z}
\end{array}\right]^{T}=\frac{B_{0}}{r^{5}}\left[\begin{array}{c}
3 X Z \\
3 Y Z \\
2 Z^{2}-X^{2}-Y^{2}
\end{array}\right]
$$

The acceleration due to the Lorentz force which experienced with the magnetic field is given by:

$$
\mathbf{a}_{m}=\frac{\mathbf{F}_{m}}{m}=\frac{q}{m}\left(\mathbf{v}_{\mathbf{r}} \times \mathbf{B}\right)
$$

where $\frac{q}{m}$ is the charge-to-mass ratio of the satellite in Coulombs per kilogram $(\mathrm{C} / \mathrm{kg})$.

The relative velocity can be written as:

$$
\mathbf{v}_{\mathbf{r}}=\left[\begin{array}{l}
v_{x} \\
v_{y} \\
v_{z}
\end{array}\right]=\left[\begin{array}{c}
\dot{r}+\dot{x}-y\left(\dot{\theta}_{z}-\omega_{E} \cos i\right)-z \omega_{E} \cos \theta \sin i \\
\dot{y}-(r+x)\left(\dot{\theta}_{z}-\omega_{E} \cos i\right)+z \omega_{E} \sin \theta \sin i \\
\dot{z}-(r+x) \omega_{E} \cos \theta \sin i+y \omega_{E} \sin \theta \sin i
\end{array}\right]
$$

Where $\omega_{E}$ is Earth's rotation rate.

Therefore, by substituting Eq. (11) and Eq. (9) into Eq. (10), the expressions of Lorentz magnetic acceleration in relative motion frame can be written as:

$$
\begin{aligned}
& a_{m x}=\frac{q B_{0}}{m r^{5}}\left[\begin{array}{l}
\left(\dot{y}-(r+x)\left(\dot{\theta}-\omega_{E} \cos i\right)+z \omega_{E} \sin \theta \sin i\right)\left(2 z^{2}-\left(x^{2}+y^{2}\right)\right) \\
-\left(\dot{z}-(r+x) \omega_{E} \cos \theta \sin i+y \omega_{E} \sin \theta \sin i\right) 3 y z
\end{array}\right] \\
& a_{m y}=\frac{q B_{0}}{m r^{5}}\left[\begin{array}{l}
\left(\dot{z}-(r+x) \omega_{E} \cos \theta \sin i+y \omega_{E} \sin \theta \sin i\right) 3 x z- \\
\left(\dot{r}+\dot{x}-y\left(\dot{\theta}-\omega_{E} \cos i\right)-z \omega_{E} \cos \theta \sin i\right)\left(2 z^{2}-\left(x^{2}+y^{2}\right)\right)
\end{array}\right] \\
& a_{m z}=\frac{q B_{0}}{m r^{5}}\left[\begin{array}{l}
\left(\dot{r}+\dot{x}-y\left(\dot{\theta}-\omega_{E} \cos i\right)-z \omega_{E} \cos \theta \sin i\right) 3 y z- \\
\left(\dot{y}-(r+x)\left(\dot{\theta}-\omega_{E} \cos i\right)+z \omega_{E} \sin \theta \sin i\right) 3 x z
\end{array}\right]
\end{aligned}
$$


We can be reformulated equation of Lorentz accelerations in case of the magnetic dipole field is tilted with respect to Earth's rotation axis fig. (1).

The magnetic field (B) has the form G.E. Pollock et al. [7]

$$
\mathbf{B}=\left[\begin{array}{lll}
B_{x} & B_{y} & B_{z}
\end{array}\right]=\frac{B_{0}}{r_{d}^{3}}\left[3\left(\hat{\mathbf{n}} . \hat{\mathbf{r}}_{d}\right) \hat{\mathbf{r}}_{d}-\hat{\mathbf{n}}\right]
$$

where $\hat{\mathbf{n}}$ is the unit vector in the direction of the magnetic dipole moment, and $\hat{\mathbf{r}}_{d}=\frac{1}{r_{d}}\left[\begin{array}{lll}r+x & y & z\end{array}\right]^{T}$

The expression of the magnetic dipole unit vector $(\hat{\mathbf{n}})$ in the LVLH frame is L.U. Si-Zhen [15]:

$$
\begin{aligned}
& \hat{\mathbf{n}}=\left[\begin{array}{lll}
n_{x} & n_{y} & n_{z}
\end{array}\right]^{T}= \\
& {\left[\begin{array}{c}
-(\cos \varepsilon \cos \theta+\sin \varepsilon \cos i \sin i) \sin \alpha-\sin i \sin \theta \cos \alpha \\
(\cos \varepsilon \sin \theta-\sin \varepsilon \cos i \cos \theta) \sin \alpha-\sin i \cos \theta \cos \alpha \\
\sin \varepsilon \sin i \sin \alpha-\cos i \cos \alpha
\end{array}\right]}
\end{aligned}
$$

where $\alpha$ is the tilt of the dipole angle between $\hat{Z}$ and $\hat{\mathbf{n}}$.

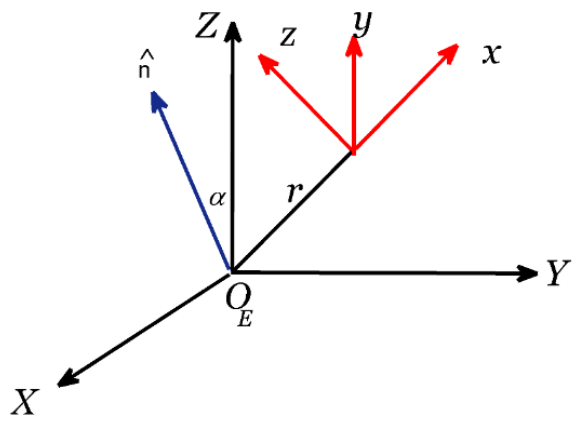

Figure 1. Earth magnetic with dipole angle $(\alpha)$

The angle $\varepsilon=\Omega_{\mathrm{m}}-\Omega$, with $\Omega_{\mathrm{m}}=\omega_{E} t+\Omega_{0}$ is the inertial rotational angle of the magnetic dipole, with $\Omega$ being the right ascension of the ascending node of the chief, and $\Omega_{0}$ is the initial rotation angle of the dipole.

The expressions of Lorentz magnetic acceleration in relative motion in case of the tilt dipole magnetic can be derived as the following:

$$
\mathbf{a}_{m}=\frac{q}{m}\left[\begin{array}{l}
a_{m x} \\
a_{m y} \\
a_{m z}
\end{array}\right]=\frac{q}{m}\left[\begin{array}{l}
v_{x} B_{z}-v_{z} B_{y} \\
v_{z} B_{x}-v_{x} B_{z} \\
v_{x} B_{y}-v_{y} B_{x}
\end{array}\right]
$$

\subsection{Lorentz Force Experience with Electric Field}

The charge distribution in a material is discrete, meaning that charge exists only where electrons and ions are and nowhere else F.T. Ulaby and U. Ravaioli [16]. In case of differential charging on satellite surface, suppose two point charges of equal magnitude but opposite polarity, separated by a distance $d$, the electric dipole is consisted, so to determine the electric potential $V_{e}$ at any point $P$ by applying equation:

$$
V_{e}=\frac{1}{4 \pi \varepsilon_{0}}\left(\frac{q}{r_{1}}+\frac{-q}{r_{2}}\right)=\frac{q}{4 \pi \varepsilon_{0}}\left(\frac{r_{2}-r_{1}}{r_{1} r_{2}}\right)
$$

where $\varepsilon_{0}=8.85 \times 10^{-12} \mathrm{C}^{2} / N . \mathrm{m}^{2}$ is the permittivity of free space and $|\mathrm{r}-\mathrm{ri}|$ is the distance between the observation point and the location of the charge $q$.

Since $\mathrm{d}<<r$ in which case the following approximations apply: $r_{2}-r_{1} \approx d \cos \Theta, r_{2} r_{1} \approx r^{2}$ L.U. Si-Zhen [16].

$$
V_{e}=\frac{q d \cos \Theta}{4 \pi \varepsilon_{0} r^{2}}
$$

where $\mathrm{d}$ is the distance vector from the charge

$(+q)$ to charge (- q) L.U. Si-Zhen [16].

In spherical coordinates, we can write the electric force as the following:

$$
\mathbf{F}_{e}=-\nabla V_{e}=-\left(\frac{\partial v_{e}}{\partial r} \hat{\mathbf{r}}+\frac{1}{r} \frac{\partial v_{e}}{\partial \Theta} \hat{\Theta}+\frac{1}{r \sin \phi} \frac{\partial v_{e}}{\partial \phi} \hat{\phi}\right)
$$

The Lorentz force experienced by an electric dipole moment in the presence of an electric field is:

$$
\begin{aligned}
& \mathbf{F}_{e}=\frac{q d}{4 \pi \varepsilon_{0} r^{3}}[2 \cos \Theta \hat{\mathbf{r}}+\sin \Theta \hat{\Theta}+0 \hat{\phi}] \\
& =\frac{q d}{4 \pi \varepsilon_{0} r^{3}}\left[\begin{array}{ccc}
\sin \Theta \cos \phi & \cos \Theta \cos \phi & -\sin \phi \\
\sin \Theta \sin \phi & \cos \Theta \sin \phi & \cos \Theta \\
\cos \Theta & -\sin \Theta & 0
\end{array}\right]\left[\begin{array}{l}
2 \cos \Theta \\
\sin \Theta \\
0
\end{array}\right]
\end{aligned}
$$

The final expressions of Lorentz acceleration Expand by the electric field in Cartesian coordinates can be derived as:

$$
\begin{gathered}
\mathbf{F}_{e}=\frac{q d}{4 \pi \varepsilon_{0} r^{5}}\left[\begin{array}{c}
3 X Z \\
3 Y Z \\
2 Z^{2}-X^{2}-Y^{2}
\end{array}\right] \\
\mathbf{a}_{e}=\left[\begin{array}{lll}
a_{e x} & a_{e y} & a_{e z}
\end{array}\right]^{T}=\frac{\mathbf{F}_{e}}{m}=\frac{q}{m} \frac{d}{4 \pi \varepsilon_{0} r^{4}}\left(\begin{array}{l}
x \\
y \\
z
\end{array}\right)
\end{gathered}
$$

$$
\begin{aligned}
& a_{e}=\left[\begin{array}{c}
a_{e x} \\
a_{e y} \\
a_{e z}
\end{array}\right]=\frac{q}{m} \frac{d}{4 \pi \varepsilon_{0} r^{4}} \\
& {\left[\begin{array}{c}
q_{1}\left(x^{2}-y^{2}\right)+q_{2} x y+s_{1} x z-s_{2} z y \\
s_{3}+s_{4} y^{2}-z^{2}+2 s_{6} y x+q_{3}\left(s_{6} z y+s_{7} z x\right) \\
-q_{4} x^{2}-q_{5} y^{2}-q_{6} z^{2}+6 q_{7} z x+6 q_{8} x y+6 q_{9} z y
\end{array}\right]}
\end{aligned}
$$

Where,

$$
\mathrm{q}_{1}=\cos \mathrm{u} \sin \mathrm{u} \sin \mathrm{i}, \quad \mathrm{q}_{2}=\sin \mathrm{i}\left(\cos ^{2} \mathrm{u}-\sin ^{2} \mathrm{u}\right)
$$




$$
\begin{array}{ll}
\mathrm{q}_{3}=\left(1-2 \sin ^{2} \mathrm{i}\right) \sin \mathrm{i} \cos \mathrm{i}, & \mathrm{q}_{4}=\left(1-\cos ^{2} \mathrm{u} \sin ^{2} \mathrm{i}\right), \\
\mathrm{q}_{5}=1-\cos ^{2} \mathrm{u}\left(1+4 \sin ^{2} \mathrm{i}\right), & \mathrm{q}_{6}=\left(1-\cos ^{2} \mathrm{i}\right), \\
\mathrm{q}_{7}=\sin \mathrm{u} \operatorname{cosi\operatorname {sin}\mathrm {i},} & \mathrm{q}_{8}=\cos \mathrm{u} \sin \mathrm{u} \sin ^{2} \mathrm{i}, \\
\mathrm{q}_{9}=\cos \mathrm{u} \operatorname{cosi\operatorname {sin}\mathrm {i},} & \mathrm{s}_{1}=\cos \mathrm{u} \cos \mathrm{i}, \\
\mathrm{s}_{2}=\sin \mathrm{u} \cos \mathrm{i}, & \mathrm{s}_{4}=\cos ^{2} \mathrm{u} \\
\mathrm{s}_{5}=\sin \mathrm{u} \cos \mathrm{u}, & \mathrm{s}_{6}=\cos \mathrm{u} \mathrm{s}_{7}=\sin \mathrm{u}
\end{array}
$$

\section{Numerical Results and Discussions}

In this section, we discuss the numerical simulations for verifying the effect of different accelerations on the relative position between two satellites using Equations (6), (12), (15) and (22). We applied our models for two different real examples formation satellite, in the first one the relative position is about $1.5 \mathrm{~km}$ (Tan DEM-X \& TERRASAR X), and the other one (CLOUDSAT\& CALIPSO) the relative position is about $100 \mathrm{~km}$. The initial orbital elements for both examples are in Tables (1), (2).

Figure 2 shows the relative position error for model nonlinear perturbed under the effect of $J_{2}$ after 10 orbital periods for two different examples. We note that in non-linear perturbed elliptical motion model can predict the relative drift caused by the $\mathrm{J}_{2}$ perturbation and shows a growing relative error in $\mathrm{x}$, and $\mathrm{y}$ axes. On the other hand, the drift error for the normal relative position increases positively about $+100 \mathrm{~m}$ after 6 hours where the nonlinear effect is primary at the beginning. However, the second-order $\mathrm{J}_{2}$ effect overwhelms the nonlinear effect after 16 hours, so, the drift error tends to the negative direction about $-700 \mathrm{~m}$.

Table 1. Orbital elements for Tan DEM-X \& TERRA SAR X Formation satellite

\begin{tabular}{|c|c|c|}
\hline \multirow{2}{*}{$\begin{array}{c}\text { Description/sat } \\
\text { ellite }\end{array}$} & \multicolumn{2}{|c|}{ First example } \\
\cline { 2 - 3 } & Tan DEM-X & TERRA SAR X \\
\hline NORAD ID: & 36605 & 31698 \\
\hline Perigee: & $514.3 \mathrm{~km}$ & $514.2 \mathrm{~km}$ \\
\hline Apogee: & $516.8 \mathrm{~km}$ & $516.9 \mathrm{~km}$ \\
\hline Inclination: & $97.4^{\circ}$ & $97.4^{\circ}$ \\
\hline $\begin{array}{c}\text { Semi-major } \\
\text { axis: }\end{array}$ & $6886 \mathrm{~km}$ & $6886 \mathrm{~km}$ \\
\hline Period: & $94.8 \mathrm{~min}$ & $94.8 \mathrm{~min}$ \\
\hline
\end{tabular}

Table 2. Orbital elements for CLOUDSAT \& CLOUDSAT Formation satellite

\begin{tabular}{|c|c|c|}
\hline \multirow{2}{*}{$\begin{array}{c}\text { Description/sat } \\
\text { ellite }\end{array}$} & \multicolumn{2}{|c|}{ Second example } \\
\cline { 2 - 3 } & CLOUDSAT & CLOUDSAT \\
\hline NORAD ID: & 29107 & 29107 \\
\hline Perigee: & $689.8 \mathrm{~km}$ & $689.8 \mathrm{~km}$ \\
\hline Apogee: & $702.2 \mathrm{~km}$ & $702.2 \mathrm{~km}$ \\
\hline Inclination: & $98.1^{\circ}$ & $98.1^{\circ}$ \\
\hline $\begin{array}{c}\text { Semi major } \\
\text { axis: }\end{array}$ & $7066 \mathrm{~km}$ & $7066 \mathrm{~km}$ \\
\hline Period: & $98.8 \mathrm{~min}$ & $98.8 \mathrm{~min}$ \\
\hline
\end{tabular}
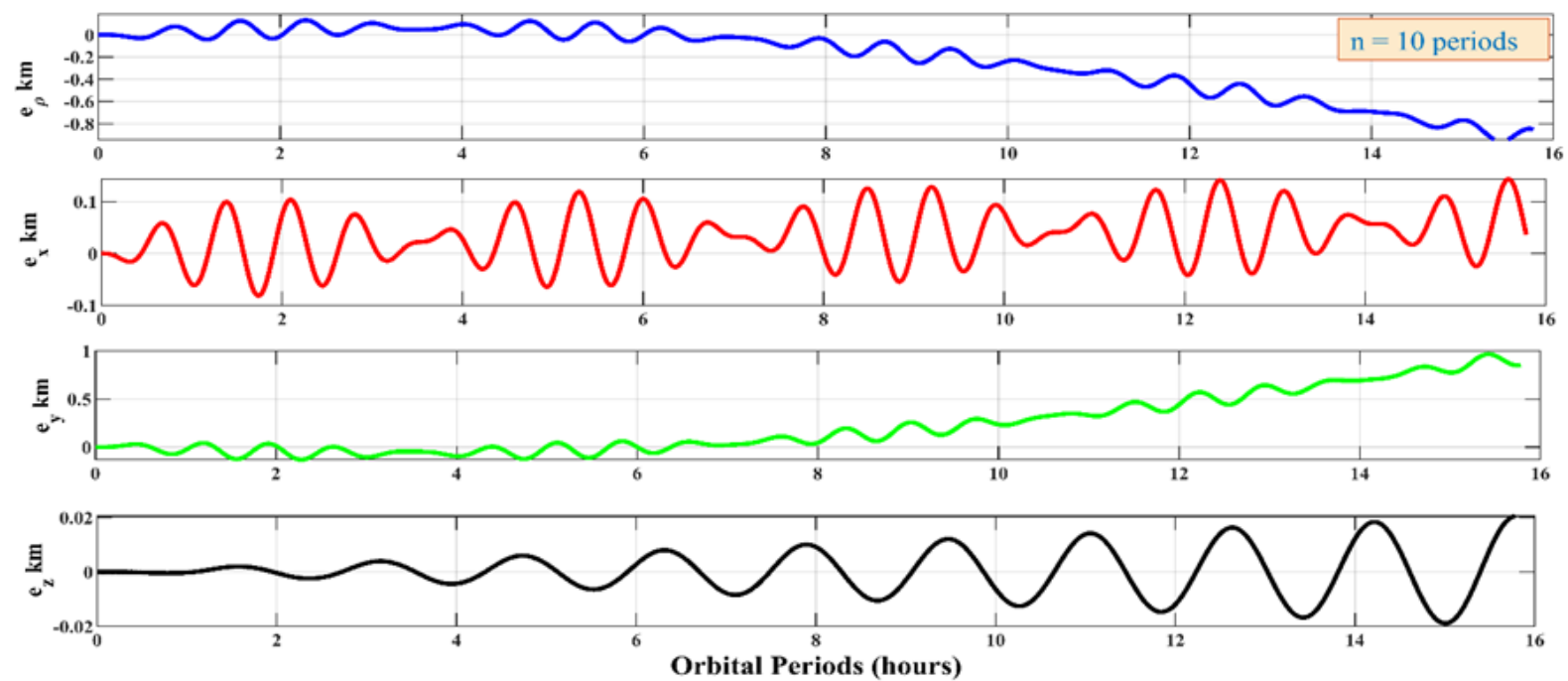

Figure 2A. The relative position drift caused by the $\mathrm{J}_{2}$ perturbation for formation flying Satellite (TanDEM-X \&TERRA SAR) 

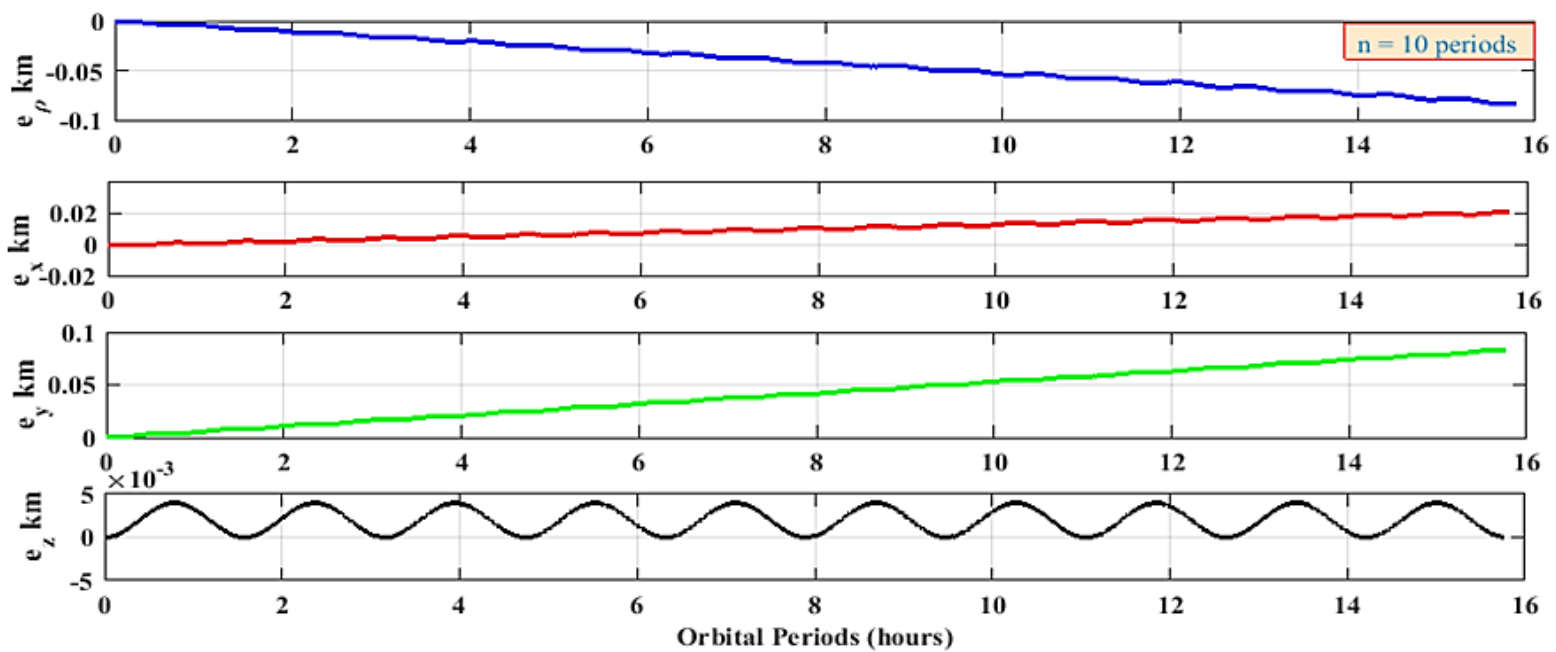

Figure 2B. The relative position drift caused by the $\mathrm{J}_{2}$ perturbation for formation flying Satellite (CLOUDSAT \& CALIPSO)
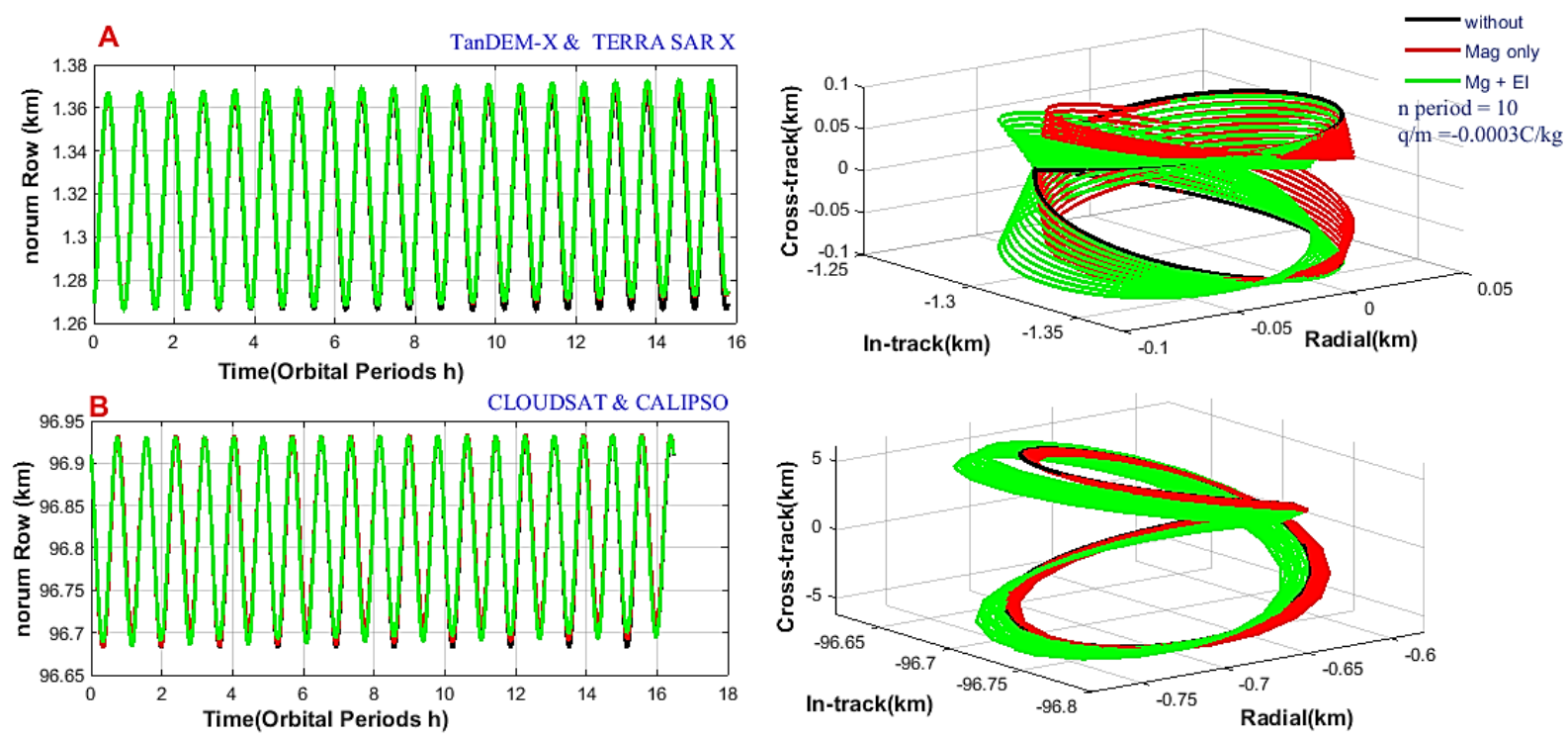

Figure 3. The drift in relative position and trajectory for magnetic and electromagnetic forces 

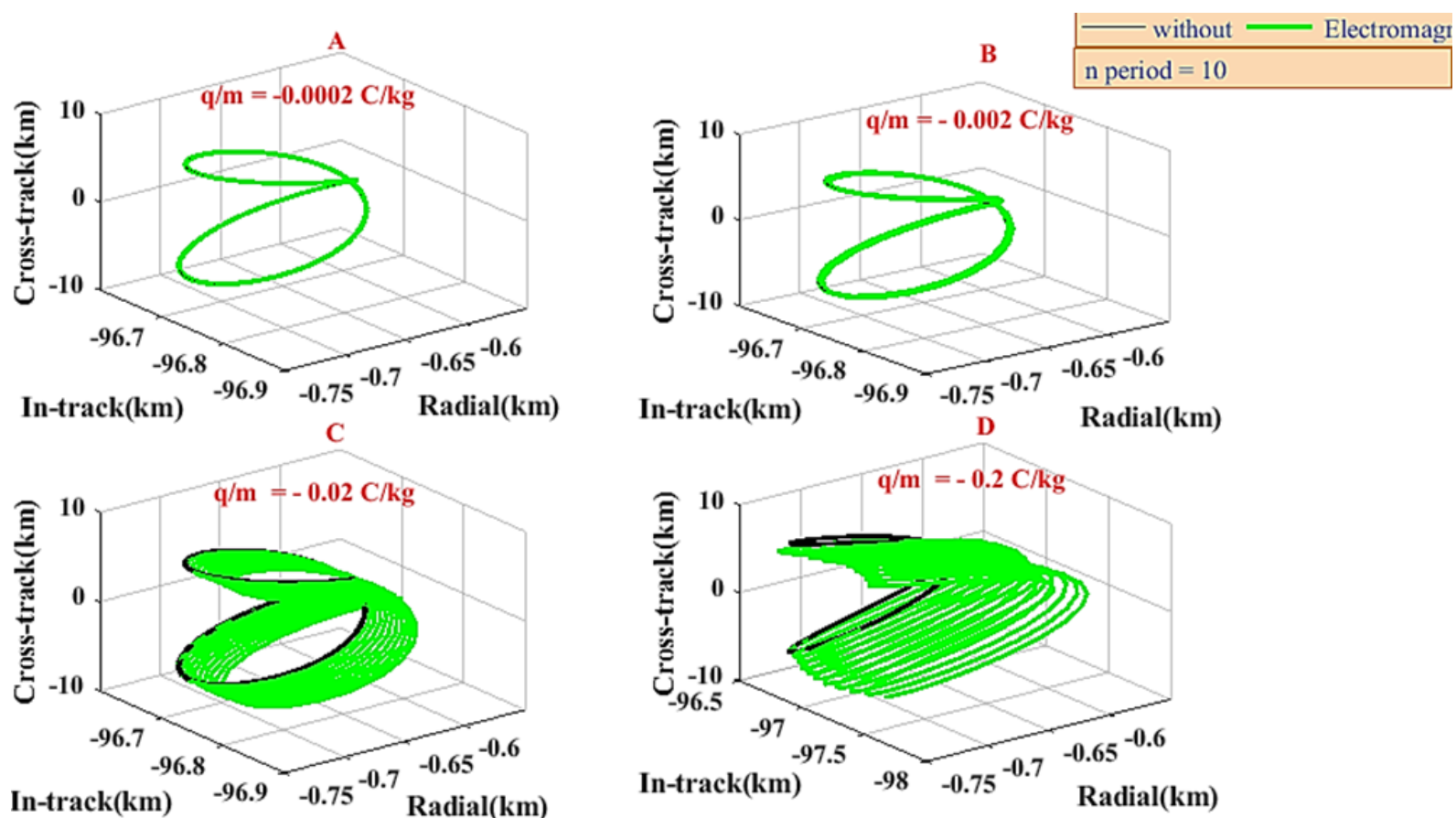

Figure 4. Relative Position trajectory with the different values of the charge to mass ratio $\mathrm{q} / \mathrm{m}$

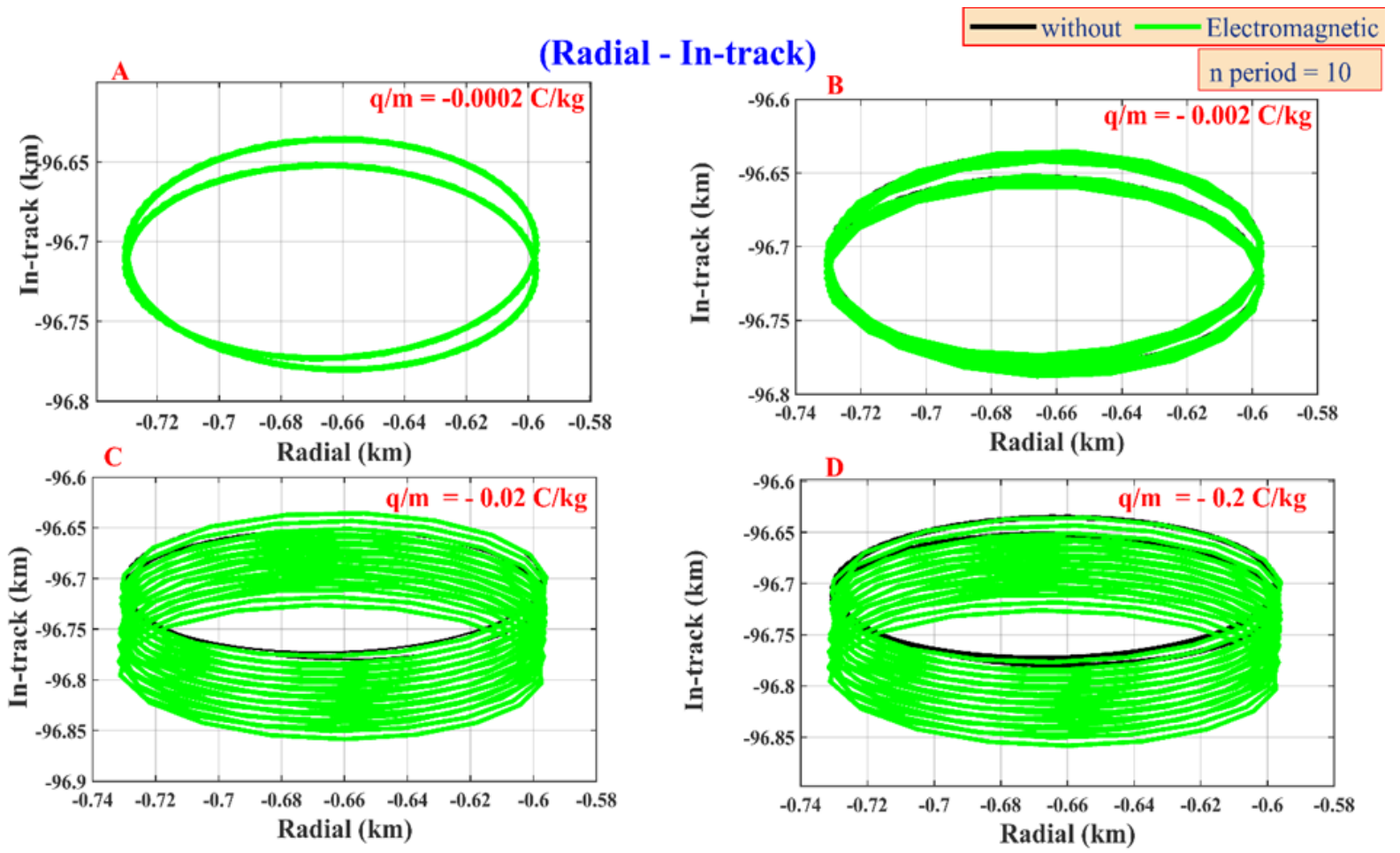

Figure 5. Radial-in-track plane for different values of the charge to mass ratio $\mathrm{q} / \mathrm{m}$ 


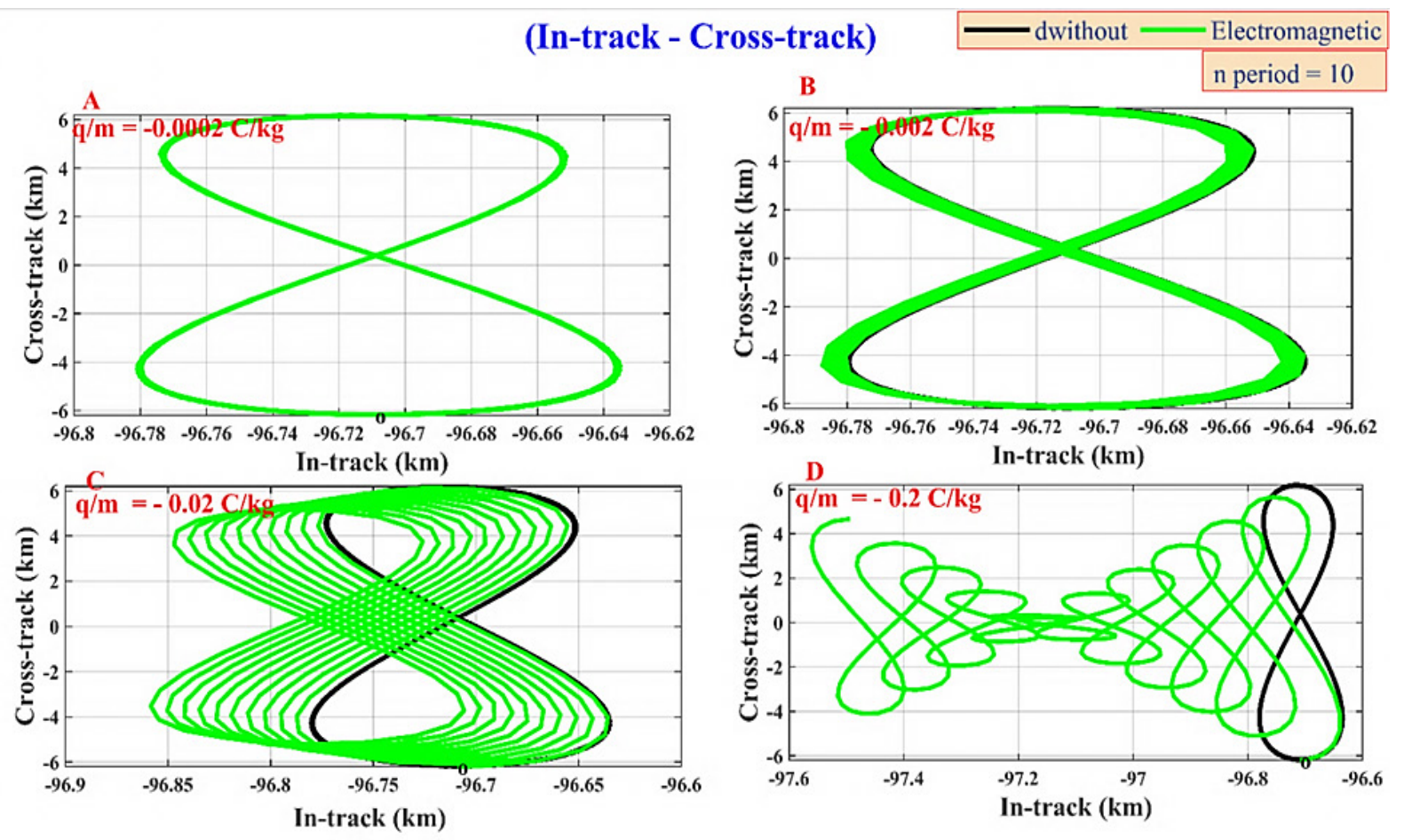

Figure 6. In-track-cross-track plane for different values of the charge to mass ratio $\mathrm{q} / \mathrm{m}$

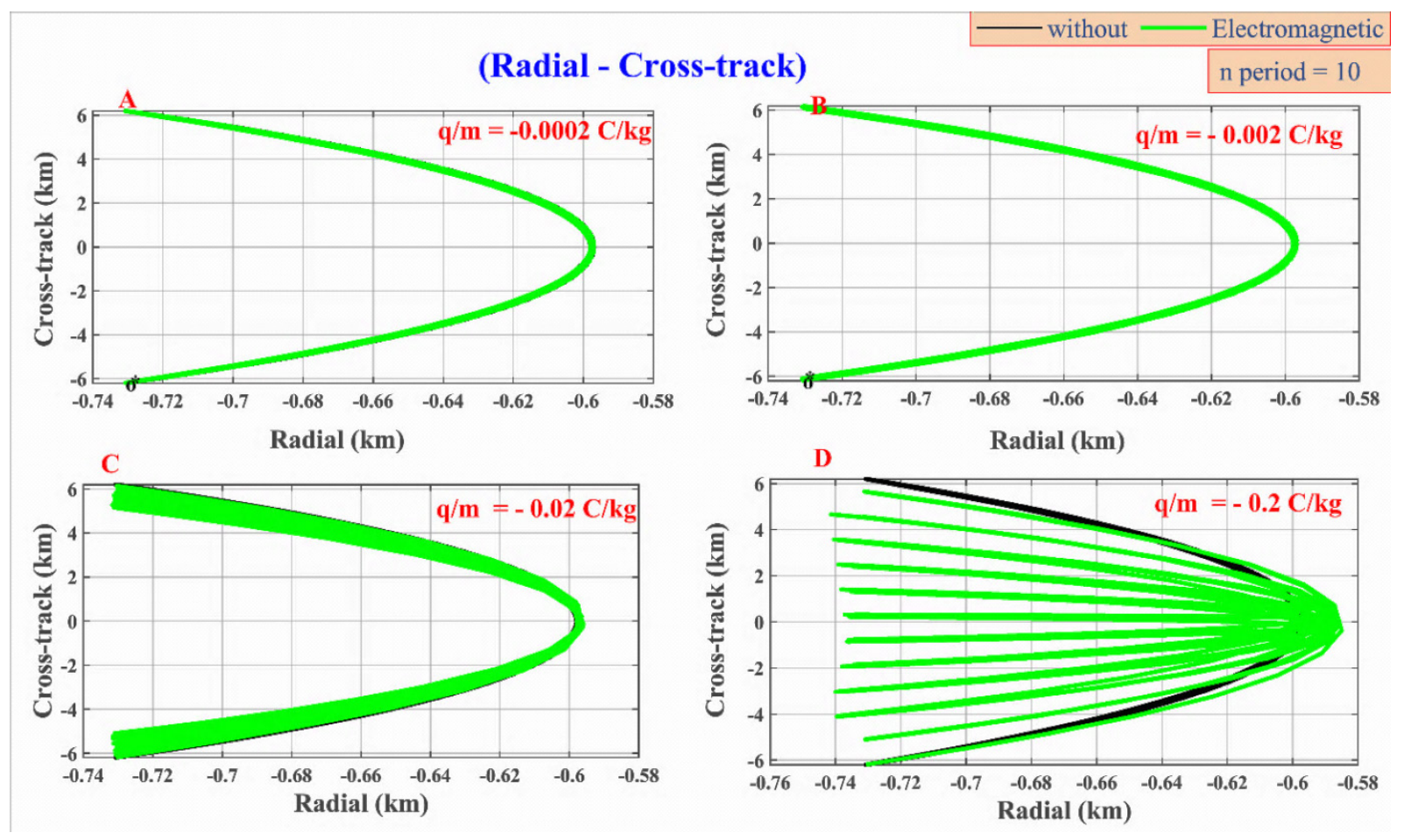

Figure 7. Radial-cross-track plane for different values of the charge to mass ratio $\mathrm{q} / \mathrm{m}$ 

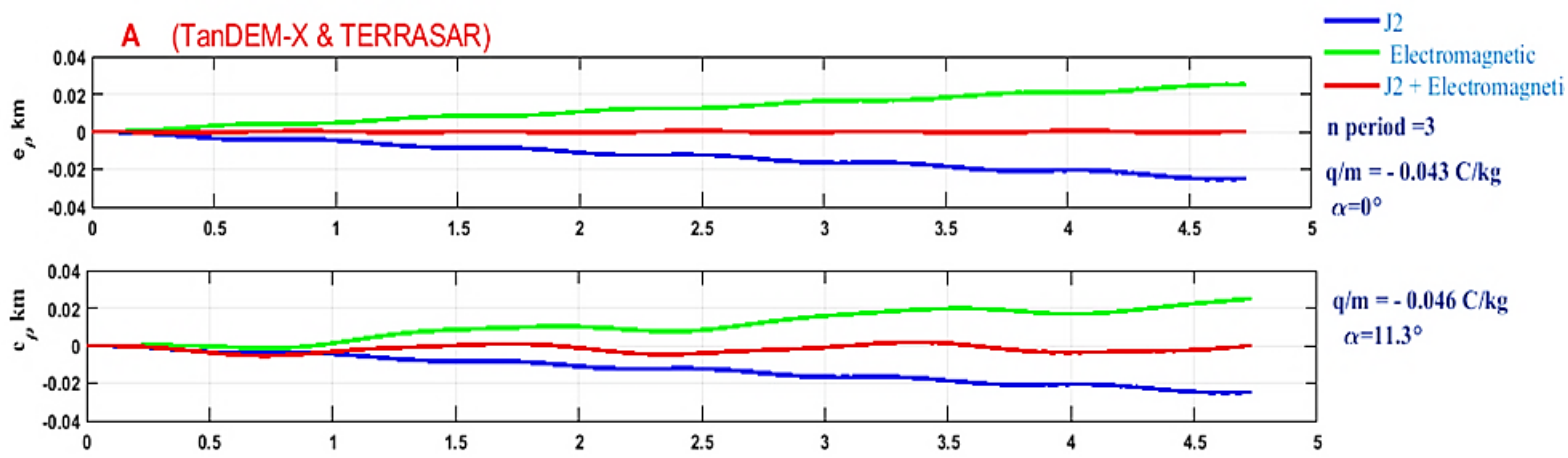

B (CLOUDSAT \&CALIPSO)

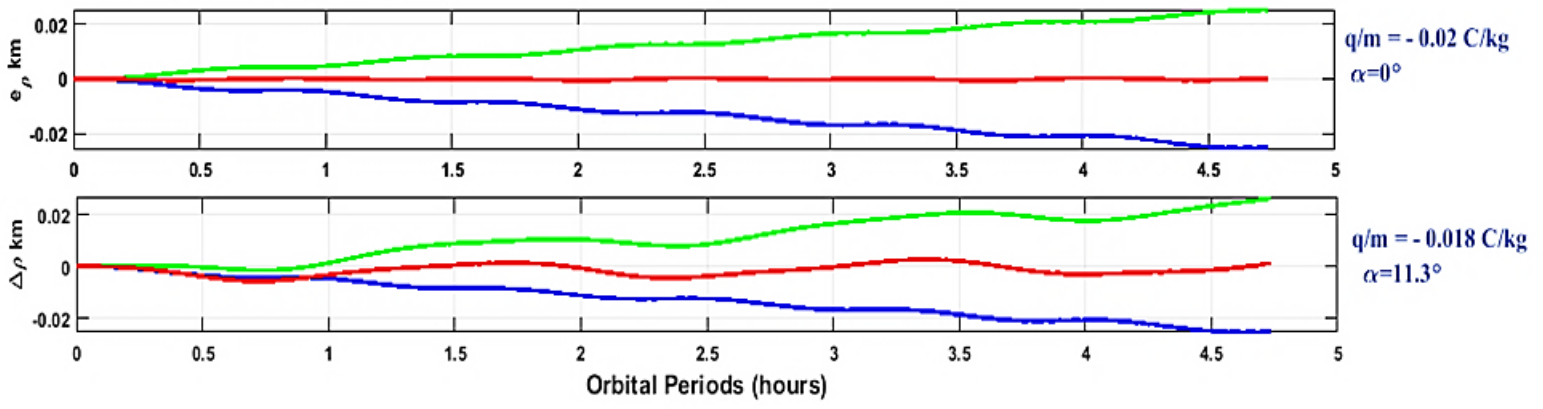

Figure 8. Correction the drift due to $\mathrm{J}_{2}$ using electromagnetic forces in relative position
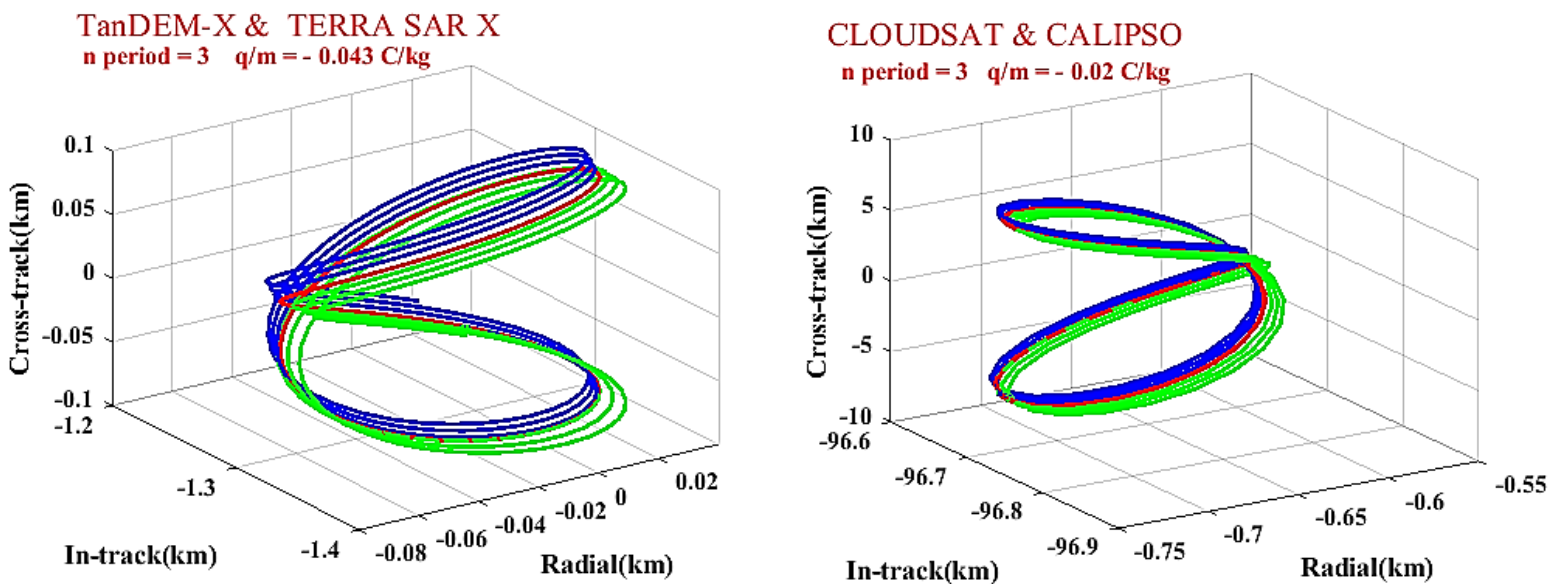

Figure 9. Correction the drift due to $\mathrm{J}_{2}$ using electromagnetic forces in the trajectory

Consider Fig. 3, which plots the drift in the relative position caused by the magnetic and electromagnetic forces, at the same value of charge to mass ratio) is $-3 \times 10^{-4}$ $\mathrm{C} / \mathrm{kg}$. From the relative trajectory case, when we used magnetic force only the change occurs in y-direction only, but by adding electromagnetic force the relative position drifts to the $x-y$ direction. On the other hand, change in the total norm relative position under the effect of electromagnetic relative position drifts to $x-y$ direction increases the accuracy of the results more than using the magnetic field only.

The effect of Electromagnetic field when we increase the level of charging in the spacecraft surface as shown in Figures $(4,5,6,7)$ for different values of charge to mass ratio $\left(2 \times 10^{-4}, 2 \times 10^{-3}, 2 \times 10^{-2}, 2 \times 10^{-1} \mathrm{C} / \mathrm{kg}\right)$ on relative trajectory, $(\mathrm{x}-\mathrm{y})$ plane, $(\mathrm{y}-\mathrm{z})$ plane, and $(\mathrm{x}-\mathrm{z})$ plane after 10 periods.

The effect of the Electromagnetic field at a charge to mass ratio $2 \times 10^{-4} \mathrm{C} / \mathrm{kg}$ will be 20 -meter change on the relative position after 10 periods, as shown in fig4-A. When the charge to mass ratio increase to $2 \times 10^{-3} \mathrm{C} / \mathrm{kg}$ as shown in fig 4-B, the change in relative position becomes within 30 meters, rises to 50 meters with $2 \times 10-2 \mathrm{C} / \mathrm{kg}$ of charge to mass ratio, as shown in fig. 4-C figure. This is a safe value of charge and enough to correct the drift in 
relative position due to second zonal harmonic $\left(\mathrm{J}_{2}\right)$ perturbation. Finally, when the charge increase to $2 \times 10^{-1}$, the value changes about 300 meters as shown in fig. 4-D. The change of trajectory position shows that the effect of increasing change appears at out-plane (z-y) clearly, but in-plane (x-y) is small change.

Maneuver and keeping formation flying need to short-duration, so we study the drift of second zonal harmonic after 3 periods. Norm relative position with time for two examples as shown in fig 8 , and relative trajectory as can be seen from Fig. 9. When neglect tilted dipole magnetic of Earth, the results showed that the value of charge to mass ratio required to correct the drift of $\mathrm{J}_{2}$ is $-0.043 \mathrm{C} / \mathrm{kg}$ for the first example and $-0.02 \mathrm{C} / \mathrm{kg}$ for the second example. On the other hand, taking into account tilted dipole magnetic $\left(\alpha=11.3^{\circ}\right)$, the value of charge to mass decreasing to correct the effect of $\mathrm{J}_{2}$ for the first example is $-0.045 \mathrm{C} / \mathrm{kg}$ and $-0.18 \mathrm{C} / \mathrm{kg}$ for the second example.

\section{Conclusions}

We developed a new approach (model) for formation flying satellites using Electromagnetic force. The Lorentz acceleration has been developed for two parts: a) the first part, which experienced with the magnetic field in the case of absolute charging of the spacecraft, including the effect of Earth tilted/Non tilted magnetic dipole; b) the second part, which is experienced with the electric field in the case of differential charging of the surface of the spacecraft. In both cases, we increase the level of charging in the spacecraft surface to produce a sufficient magnitude of Lorentz force to be capable of controlling spacecraft (orbit or attitude). Numerical results have shown that the accuracy of the Lorentz force has increased to correct the relative position at a level of $2 \mathrm{~m}$ after included both magnetic and electric fields after three orbital periods. We have applied Our model to studied the Lorentz acceleration for spacecraft formation flying control to correct the drift in relative position due to second zonal harmonic $\left(\mathrm{J}_{2}\right)$. The numerical results shown that the value of the charge to mass ratio is about \pm 0.02 to $\pm 0.04 \mathrm{C} / \mathrm{kg}$ can be valid to correct drift in relative position. In future work, we are going to use feedback control for optimal control of spacecraft formation flying using electrostatic forces.

\section{REFERENCES}

[1] B. Kuiack and S. Ulrich. Nonlinear Analytical Equations of Relative Motion on J 2-Perturbed Eccentric Orbits. Journal of Guidance, Control, and Dynamics, 41(12), pp.2666-2677, 2018

[2] M. Bakhtiari, K. Daneshjou and M. Fakoor. Long-term effects of main-body's obliquity on satellite formation perturbed by third-body gravity in elliptical and inclined orbit. Research in Astronomy and Astrophysics, 17(4), p. 039, 2017.

[3] D. Vokrouhlicky. The geomagnetic effects on the motion of an electrically charged artificial satellite. Celestial Mechanics and Dynamical Astronomy, 46(1), pp. 85-104, 1989.

[4] M. Peck. August. Prospects and challenges for Lorentz-augmented orbits. In AIAA guidance, navigation, and control conference and exhibit (p. 5995), 2005.

[5] Y. A. Abdel - Aziz, February. Lorentz force effects on the orbit of a charged artificial satellite: a new approach. In AIP Conference Proceedings (Vol. 888, No. 1, pp. 385-391).AIP, 2007.

[6] Y. A. Abdel-Aziz, and K.I. Khalil, Electromagnetic effects on the orbital motion of a charged spacecraft. Research in Astronomy and Astrophysics, 14(5), p.589. 2014.

[7] G.E. Pollock, J.W. Gangestad and J.M. Longuski. Analytical solutions for the relative motion of spacecraft subject to Lorentz-force perturbations. Acta Astronautica, 68(1-2), pp. 204-217. 2011.

[8] S. Tsujii, M. Bando and H. Yamakawa. Spacecraft formation flying dynamics and control using the geomagnetic Lorentz force. Journal of Guidance, Control, and Dynamics, 36(1), pp. 136-148. 2012.

[9] X. Huang, Y. Yan, Y. Zhou and T. Yi. Improved analytical solutions for relative motion of Lorentz spacecraft with application to relative navigation in low Earth orbit. Proceedings of the Institution of Mechanical Engineers, Part G: Journal of Aerospace Engineering, 228(11), pp. 2138-2154. 2014

[10] C. Peng, and Y. Gao. Formation-Flying Planar Periodic Orbits in the Presence of Inter satellite Lorentz Force. IEEE Transactions on Aerospace and Electronic Systems, 53(3), pp. 1412-1430. 2017.

[11] G. Y. Xu, and D. W. Wang. Nonlinear Dynamic Equations of Satellite Relative Motion around an Oblate Earth," Journal of Guidance, Control, and Dynamics, Vol. 31, No. 5, pp. $1521-1524.2008$

[12] H.D. Curtis. Orbital mechanics for engineering students. Butterworth-Heinemann. 2013.

[13] J.S. Ginn. Spacecraft Formation Flight: Analysis Of The Perturbed J2-modified Hill-Clohessy-Wiltshire Equations. 2007.

[14] F. T. Ulaby. Electromagnetics for Engineers (Vol. 1). Prentice Hall, 2005.

[15] L. U. Si-Zhen, L. U. Cao, X. Y. ZHANG, and Q.I.N. Xiao-Yong. Fuel-optimal Lorentz-augmented Spacecraft Formations Using Novelty Minimum Sliding Mode Error Feedback Controller. DEStech Transactions on Computer Science and Engineering, (aice-ncs), 2016.

[16] F. T. Ulaby and U. Ravaioli. Fundamentals of Applied Electromagnetics, 7/E. 2015. 